Revue européenne des sciences sociales

European Journal of Social Sciences

XLIV-134 | 2006

Quel(s) défi(s) pour les sciences sociales à l'heure de la mondialisation?

\title{
Des Argonautes aux internautes
}

Marie-Dominique Perrot et Gilbert Rist

\section{(2) OpenEdition}

\section{Journals}

Édition électronique

URL : http://journals.openedition.org/ress/301

DOI : 10.4000/ress.301

ISSN : 1663-4446

Éditeur

Librairie Droz

\section{Édition imprimée}

Date de publication : 1 juin 2006

Pagination : 203-214

ISBN : 9-782-600-01095-5

ISSN : 0048-8046

Référence électronique

Marie-Dominique Perrot et Gilbert Rist, " Des Argonautes aux internautes », Revue européenne des sciences sociales [En ligne], XLIV-134 | 2006, mis en ligne le 14 octobre 2009, consulté le 01 mai 2019. URL : http://journals.openedition.org/ress/301; DOI : 10.4000/ress.301 


\section{Marie-Dominique PERROT Gilbert RIST}

\section{DES ARGONAUTES AUX INTERNAUTES}

Il s'agit, modestement, de risquer une hypothèse à propos de l'unité et de la diversité dans les travaux de Gérald Berthoud. Comme cela est fréquent, à première vue, la diversité l'emporte sur l'unité. Il y eut d'abord les années passées à explorer l'échange et le don - de Mauss à Polanyi - et l'étude des pratiques «traditionnelles» pour retrouver «l'archaïque» (considéré comme un fondement toujours actuel et nécessaire) puis, plus récemment, l'intérêt pour les NTIC et la société de l'information, qui - étrangères au «roc»sur lequel s'établit le lien social - nous font accéder à un monde virtuel. Apparent mystère d'une double démarche qui conjugue le plus «primitif» et le plus (post)moderne, une anthropologie des plus classiques et l'étude d'une société en voie de formation, s'il faut en croire les augures technocratiques...

Aux oppositions, à la «polarité», à l'affirmation de la diversité, il convient toujours - comme Berthoud ne cesse de le répéter - de prendre en compte les similitudes, l'analogie, la recherche de l'unité. L'injonction s'applique bien entendu à la comparaison entre sociétés, afin d'éviter la tentation du «grand partage» aussi bien que celle du réductionnisme sociocentrique. Peut-elle concerner les intérêts de recherche d'une personne particulière (on n'ose écrire d'un «individu » singulier...)? Même si cette approche reste spéculative, on peut néanmoins risquer le pari.

Il y a probablement quelque outrecuidance à comparer les échanges ritualisés des Trobriandais avec le partage des biens informationnels que pratiquent les nomades sédentaires qui habitent le «village global». N'appartiennent-ils pas à deux sociétés que tout oppose? L'une fermement ancrée dans le pôle que l'on dit «traditionnel», et dans un lieu géographique déterminé, l'autre marquée par la modernité ou la «surmodernité », affranchie des contraintes de l'espace et revendiquant l'ubiquité. On pourrait peut-être tenter de justifier la démarche à partir de l'anthropologie structuraliste qui nous a appris à produire du sens en pensant ensemble les contraires ou les oppositions, mais ce serait oublier que les écarts significatifs ne sont repérables qu'à l'échelle d'un même groupe social, ou d'un même récit.

Ces remarques visent simplement à mettre en évidence le caractère très hypothétique de ce texte, même s'il repose sur une conviction profonde, à savoir que les sociétés - même les plus distantes dans le temps ou dans l'espace - ne sont jamais totalement étrangères les unes aux autres. Certes, «les temps changent» (et les traditions aussi!), mais le discours récurrent qui annonce l'apparition d'une 
«nouvelle ère» ne saurait être crédible ${ }^{1}$. Il ressortit en effet à une tradition rhétorique qui, en isolant un fait (un événement, une technique) par rapport à l'ensemble des pratiques sociales, cherche à le faire passer pour la marque d'une solution de continuité qui affecterait la société tout entière. On sait pourtant qu'il n'en est rien: les véritables changements sont ceux dont nous n'avons pas conscience $^{2}$ plutôt que ceux que l'on annonce à son de trompe. Mais il y a plus : toutes les sociétés humaines sont confrontées à la nécessité de produire du lien social, d'inventer les moyens de prévenir le surgissement de la violence, de structurer les rapports sociaux, de faire admettre des «classements » ou des hiérarchies, en dépit d'idéologies égalitaires. Paradoxalement, la construction de la société a toujours pour effet de produire l'illusion de sa naturalité: ce qui va de soi, et qui est à la fois in-su et non-dit. Le respect de ces règles est d'autant plus obligatoire que celles-ci sont implicites (et donc indiscutables) et parce que, au bout du compte, leur respect est la condition d'une certaine permanence sociale, c'est-àdire le maintien de «l'être ensemble $»^{3}$.

Nul doute que, dans cette architecture symbolique générale, l'échange tienne une place fondamentale, parce qu'il constitue un fait social total, parce qu'il structure les relations entre les personnes et définit leurs obligations mutuelles. Certes, on s'empressera de faire remarquer qu'il y a «échange» et «échange» et qu'il ne faut pas confondre sa forme «noble» (le don) avec sa forme marchande où le bien l'emporte sur le lien. Or, même dans ce cas extrême - que l'on pourrait considérer comme le degré zéro de l'échange - on peut néanmoins admettre qu' "il se passe quelque chose » (de l'ordre de la sociabilité minimale) entre le vendeur et l'acheteur, même si ce «quelque chose» est réduit à l'instant. Comme Adam Smith le faisait déjà remarquer: "On n'a jamais vu un chien faire de propos délibéré l'échange d'un os avec un autre chien ${ }^{4}$.

A partir de là, si l'on s'intéresse aux objets échangés plutôt qu'aux échangistes, on peut imaginer qu'ils n'ont que deux destinations possibles: la circulation ou la consommation (ou la consumation). Mais cette affirmation est trompeuse car elle ne s'attache qu'à la matérialité des biens qui prévaut dans l'échange marchand. En effet, même un repas (ou un potlatch), consommé (ou consumé) doit être rendu. On peut donc affirmer que la circulation est constitutive de l'échange.

Gérald Berthoud, Frédéric Ischy, Olivier Simioni, La société de l'information: La nouvelle frontière?, «Pratiques et théories des sciences et des techniques», VII, Lausanne, Institut d'anthropologie et de sociologie, 2002, p. 27.

Aussi bien dans l'évolution du langage que dans celle de l'environnement: il suffit d'écouter une émission radiophonique enregistrée voici 50 ans ou de comparer deux états successifs d'un même paysage pour prendre la mesure des changements qui se sont produits de manière imperceptible.

Ce qui revient à reformuler la définition durkheimienne de la religion, selon laquelle les rites associés aux croyances «sont la chose du groupe et en font l'unité» (Emile Durkheim, Les formes élémentaires de la vie religieuse. Le système totémique en Australie, Paris, Quadrige, PUF, 1985 [1912], p. 60). Dans La mythologie programmée (Marie-Dominique Perrot, Gilbert Rist, Fabrizio Sabelli, Paris, PUF, 1992), nous avions déjà montré comment certaines pratiques de la société contemporaine étaient rendues acceptables, légitimes et nécessaires en «recyclant» des bribes de matériaux tirés du fonds mythologique et religieux occidental.

4 Adam Smith, Recherches sur la nature et les causes de la richesse des nations, Gallimard, Paris, 1976 [1776], p. 47. 
Il serait peut-être possible d'identifier, sinon d'autres «invariants de l'esprit humain» (Lévi-Strauss), du moins d'autres pratiques qui fondent la vie sociale. Les croyances durkheimiennes ou le sacrifice ${ }^{5}$ pourraient être considérés comme d'autres faits sociaux totaux «qui mettent en branle l'ensemble de la société», comme disait Mauss, et qui peuvent être abordés comme des révélateurs de la fabrique sociale. Mais l'intention de ce texte n'est pas de passer en revue l'ensemble de ces pratiques transversales, communes à toutes les sociétés, mais de montrer comment l'échange et la circulation (qui sous-tendent ce que Malinowski appelait le «kula ring») peuvent permettre de porter un nouveau regard sur certaines pratiques sociales que l'on peut découvrir aujourd'hui sur la Toile. Un des rôles de l'anthropologie n'est-il pas de «nous faire mirer en la face d'autruy », selon la belle expression de Chauveton, reprise par Berthoud ${ }^{6}$ ?

\section{QUELQUES REMARQUES SUR LA KULA}

Soit donc la kula - que l'on considérera ici comme une forme emblématique de l'échange - telle que la présente Malinowski. Ses grands traits sont si connus que nous nous bornerons à en rappeler l'essentiel. Sur le chapelet d'îles que forme l'archipel des Trobriand, la principale activité des chefs consiste à échanger, au cours d'importantes cérémonies, deux sortes d'objets de prestige (vaygu'a): de très longs colliers de coquillages rouges (soulava) qui circulent dans le sens des aiguilles de la montre, et des brassards de coquillages blancs (mwali), qui circulent en sens inverse, de sorte que les partenaires de l'échange - qui sont liés entre eux par des relations stables qui peuvent durer toute une vie - ne peuvent que rendre des brassards à ceux dont ils ont reçu des colliers et donner des colliers à ceux dont ils ont reçu des brassards, puisqu'on ne reçoit jamais de la même personne à la fois des colliers et des brassards. Ces échanges se font à l'occasion de vastes expéditions maritimes, précédées de la construction des canots. En marge de ces échanges cérémoniels, et des rites magiques qui les accompagnent, se déroule une forme de véritable commerce, ou d'âpre marchandage, qui concerne les biens d'usage (gimwali).

A partir de ce rappel, plusieurs remarques s'imposent. Premièrement, pour que le système fonctionne, il faut que les vaygu' a circulent et qu' ils ne soient pas

5 Gérald Berthoud a bien montré comment le thème du sacrifice était présent dans notre société, qui en a détourné le sens pour assurer la survie du système capitaliste (Cf. «Règle de l'intérêt et nécessité du sacrifice», A quoi bon (se) sacrifier? Revue du M.A.U.S.S., n 5, 1995, p. 101-117).

` Gérald Berthoud, Vers une anthropologie générale. Modernité et altérité, Genève, Droz, 1992, p. 11.

Nous nous en tenons pour l'essentiel à la description de Malinowski (Argonauts of the Western Pacific, New York, London, Routledge \& Kegan Paul Ltd, 1964 [1922]), même si elle a été «revisitée» et critiquée notamment par Annette Wiener: «The Reproductive Model in Trobriand Society », Bulletin du M.A.U.S.S., n 2, 1982, p. 7-18; ainsi que dans Inalienable Possessions. The Paradox of Keeping-While-Giving, Berkeley, Los Angeles, Oxford, Universty of California Press, 1992. Cf. aussi J. E. Leach, The Kula: New Perspectives on Massim Exchange, Cambridge, New York, Cambridge University Press, 1983; et Frederick H. Damon, «Kula Valuables: The Problem of Value and the Production of Names », L'Homme, n 162, 2002, p. 107-136. 
accaparés par leurs détenteurs du moment. La remise en jeu périodique des colliers et des brassards constitue donc un des véritables enjeux de la kula puisque chacun a intérêt à conserver pour soi un objet dont le «prestige incorporé» retombe sur son possesseur (en avivant les distinctions sociales), tout en sachant que ce n'est qu'en activant la circulation qu'il peut espérer en obtenir un nouveau, plus prestigieux encore ${ }^{8}$. Chacun met donc en place des stratégies subtiles pour inciter son partenaire (ou l'un de ses partenaires) à céder ce dont il dispose en lui offrant des objets d'une valeur jugée suffisante pour le décider à se défaire de l'objet convoité.

Deuxièmement, la circulation accrô̂t la valeur des biens, même si ceux-ci ne sont en principe pas modifiés par leur détenteur provisoire (les colliers - parfois longs de deux à trois mètres - et les brassards sont si imposants qu'ils ne peuvent en aucun cas être portés comme objets «décoratifs» et ne peuvent être destinés qu'à l'exposition ou l'ostentation périodique, lors de grandes fêtes). En effet, chaque vaygu' a tire sa valeur du prestige reconnu à ses détenteurs successifs. Plus ceux-ci auront été nombreux (plus l'objet aura circulé), plus cette valeur sera grande.

Troisièmement, en considérant le système dans son ensemble, les vaygu'a peuvent être considérés comme une sorte de propriété collective, composée de biens inaliénables et inappropriables privativement, «entreposés» chez des possesseurs temporaires'. Ce qui explique le paradoxe - souligné par Annette Wiener - qui consiste à donner tout en conservant : «the motivation for reciprocity is centered not on the gift per se but on the authority vested in keeping inalienable possessions $»^{10}$. Malinowski - qui prend bien soin de noter que, finalement, seul l'anthropologue a une vision d'ensemble de la kula car «not even the most intelligent native has any clear idea of the kula as a big, organised construction, still less its sociological function and implication ${ }^{11}-$, ne semble pas avoir perçu cet aspect de propriété collective. Sans doute parce que les rivalités entretenues par les chefs pour obtenir des vaygu'a les plus prestigieux possibles obnubilaient le fait que leur ensemble constituait une sorte de «bien commun». Pour le dire dans le langage des économistes, les participants à la kula ont constitué, «à partir d'éléments apparemment rivaux, un ensemble non rival d'éléments circulants $»^{12}$.

${ }^{8}$ La vitesse de circulation des vaygu'a fait l'objet d'un débat. Alors que Malinowski affirme que «Thus no man ever keeps away any of the articles [soulava and mwali] for any length of time in his possession » (op. cit., p. 81), Annette Wiener affirme au contraire que «a kula player who is able to obtain a famous shell tries to keep it out of circulation, guarding it for ten, fifteen or even twenty years [...] even though eventually the shell must reenter the kula exchange» (Inalienable Possessions, op. cit., p. 133).

9 A la différence des bijoux de famille, considérés comme inaliénables - même s'il peut arriver que certains soient vendus - et qui sont transmis sous forme de dons d'une génération à l'autre, les vaygu'a ne peuvent circuler que "latéralement», à l'intérieur du cercle de la kula en stimulant la remise de contre dons. Les uns comme les autres sont d'autant plus prestigieux qu'ils témoignent d'une plus longue histoire, dont ils contribuent à entretenir la mémoire.

${ }^{10}$ Annette Wiener, op. cit., p. 40.

${ }^{11}$ Bronislaw Malinowski, op. cit., p. 83.

${ }_{12}$ Michel Gensollen, «Economie non rivale et communautés d'information», Réseaux, n 124 , 2004 , p. 184. Sur la distinction entre propriété et possession, cf. Rolf Steppacher, «La petite différence et ses grandes conséquences: possession et propriété», Brouillons pour l'avenir. Contributions au débat sur les alternatives. Nouveaux Cahiers de l'IUED, n 14, 2003, p. 181-191. 
Quatrièmement, ces échanges sont asynchrones, c'est-à-dire que l'échange des brassards et des colliers ne se fait jamais de manière simultanée. Ce qui semble par ailleurs une règle bien établie: en dehors de l'échange marchand, qui suppose un règlement immédiat, le contre don est nécessairement différé. Comme le disait déjà Tamati Ranaipiri, entre le don et son retour, «le temps passe et passe $»^{13}$. Par ailleurs, une des caractéristiques de ces biens est qu'ils ne disparaissent pas, suite à la transaction, puisqu'ils continuent de participer du circuit de l'échange.

Cinquièmement, les biens qui entrent dans le cercle de la kula ont ceci de particulier qu'ils échappent à la marchandisation et ne peuvent être utilisés comme moyen de paiement ou de contre-valeur pour d'autres biens. La séparation entre les vaygu' $a$ et les objets qui peuvent faire l'objet de transactions marchandes - par exemple les ignames - est nette et s'étend même à la manière de se comporter dans l'échange: on méprise en effet celui qui confond l'échange kula avec le marchandage lié aux gimwali.

Sixièmement, ces échanges créent du lien social entre les partenaires même si seuls les chefs - que l'on qualifierait aujourd'hui de membres de l'«élite» - ont accès à la kula. Fait remarquable, ces relations permettent de réunir des membres de tribus géographiquement distantes, qui ne partagent ni la même langue, ni les mêmes coutumes. Toutefois, ce lien social n'implique aucune intimité particulière entre les échangistes. Certes, ils se connaissent personnellement, ils savent également le nom des partenaires plus éloignés qui furent naguère les détenteurs d'un vaygu' a renommé, mais ils n'ont pas d'activité commune: il ne vont pas ensemble à la pêche, même s'ils se considèrent l'un l'autre comme amis et que chacun se sent responsable de son hôte lorsque celui-ci lui rend visite. De plus, comme chaque «joueur» de la kula dispose, selon son rang, de plusieurs «correspondants » qu'il peut privilégier selon ce qu'il attend de chacun, les chemins que peut emprunter tel ou tel objet ne sont jamais définis par avance. A, qui espérait recevoir de $\mathrm{B}$ tel collier très convoité parce que chargé d'histoire, peut très bien être déçu si $\mathrm{B}$ le transmet à $\mathrm{C}$, qui ne fait pas partie du groupe de partenaires attitrés de A. Ce qui ajoute aux rivalités puisque chacun est libre de transmettre ce qu'il possède provisoirement à des partenaires multiples. Enfin, la kula - considérée comme une institution globale - crée également du lien social entre les membres d'une même tribu, qui participent aux travaux collectifs, notamment à la construction des canots, et sont ensuite « récompensés» par leur chef ${ }^{14}$.

\footnotetext{
${ }^{13}$ Selon la traduction du texte maori proposée par Bruce Briggs et citée in Marshall Sahlins, Age de pierre, âge d'abondance. L'économie des sociétés primitives, Paris, Gallimard, 1976 [1972], p. 202-203.

${ }^{14} \ll[\ldots]$ the whole tribal life is permeated by a constant give and take. [...] wealth, given and taken, is one of the main instruments of social organisation, of the power of the chief, of the bonds of kinship and of the relations in law.» (Malinowski, op. cit., p. 167). Ou encore: «[There is a] deep tendency to create social ties through exchange of gifts. Apart from any consideration as to whether the gifts are necessary or even useful, giving for the sake of giving is one of the most important features of Trobriand sociology.» (ibid., p. 175).
} 


\section{Y AURAIT-IL DES FORMES MODERNES DE LA KULA?}

A partir de ces quelques remarques, est-il possible de discerner dans notre monde moderne - et tout particulièrement parmi les internautes - des traces de pratiques comparables ou des initiatives qui leur ressemblent? Pour cela, il convient de réduire le champ de l'enquête puisqu'il n'est pas question d'embrasser ici l'ensemble des usages repérables sur la Toile. Prétendre y parvenir serait d'ailleurs le signe d'une ambition si démesurée qu'elle ne pourrait que conduire à l'échec. De plus, ce qui est le mieux connu est souvent ce qui est le plus éloigné historiquement et géographiquement -, alors que ce qui est à la fois proche et contemporain ne nous apparaît que confusément, tant les pratiques semblent diverses et dépourvues de cohérence. Peut-être sommes-nous dans la position des indigènes dont parlait Malinowski qui, bien qu'intelligents, sont incapables d'avoir une vision d'ensemble du monde social auquel ils appartiennent... ${ }^{15}$

Ces précautions prises, écartons donc tous les sites - sans doute la majorité qui proposent la vente de biens et services (des billets d'avion et des ouvrages d'anthropologie aux images pornographiques en passant par les produits de consommation courante livrés à domicile ou les biens immobiliers), les sites d'information (des administrations, des universités, des compagnies de transport ou des moteurs de recherche) qui sont parmi les plus fréquentés, ainsi que les innombrables courriels qui s'échangent quotidiennement à travers des réseaux que l'on peut qualifier de personnels. Bien que ce premier tri puisse sans doute être plus sévère encore, il permet toutefois de faire apparaitre ces formes sociales nouvelles que sont les «communautés virtuelles», caractérisées par l'échange de biens informationnels.

Certains sont assez sceptiques par rapport à l'existence de cette nouvelle forme de lien social $^{16}$, d'autant plus que l'abus du terme «communauté » n'est plus à démontrer. Il est évident que les internautes qui débattent ensemble de sujets qui les passionnent ou qui partagent leurs connaissances pour améliorer les logiciels libres ne constituent pas une «communauté » au sens canonique du terme, mais les Argonautes - c'est-à-dire l'ensemble des chefs engagés dans la kula - ne formaient pas non plus un groupe spécifique, dont les membres étaient impliqués dans une vie quotidienne commune. Les uns comme les autres appartiennent donc à un «collectif» et sont liés par des pratiques et des intérêts communs: on peut donc considérer le terme «communauté » comme impropre, tout en reconnaissant que des liens sociaux réels peuvent se tisser dans de telles circonstances.

Sans doute faut-il encore préciser et nuancer. Nous ne prétendons pas que la simple existence de réseaux informatiques suffise à créer, ipso facto, une "société de communication» comme le pensait Norbert Wiener, ni que la manière dont Howard Rheingold définit les «communautés virtuelles ${ }^{17}$ permette d'identifier

${ }^{15} \mathrm{Cf}$. ci-dessus, note 11.

${ }^{16}$ Notamment Gérald Berthoud et al., La société de l'information, op. cit., p. 44-45.

${ }^{17}$ «Les communautés virtuelles sont des regroupements socioculturels qui émergent du réseau lorsqu'un nombre suffisant d'individus participent à ces discussions publiques pendant assez de temps en y mettant suffisamment de cœur pour que des réseaux de relations humaines se tissent au sein du Cyberespace» (Howard Rheingold, Les communautés virtuelles, Addison-Wesley France, Paris, 1995, p. 6). Il est en effet impossible de savoir ce que les termes «assez de temps», «suffisant» et «suffisamment » impliquent. 
des ensembles significatifs. Nombreux sont en effet les agrégats de personnes qui pratiquent la «communication électronique de groupe», qu'il s'agisse de jeux de rôle, de forums de discussion ou de chats (ou «espaces de clavardage » comme disent les Québécois!) ${ }^{18}$, afin de se divertir ou développer des relations interpersonnelles, quitte à se dissimuler sous des identités multiples. Or ces groupes sont souvent éphémères et leurs membres trop instables pour qu'on puisse les comparer à des «collectifs » qui partagent, sur la durée, des intérêts et des préoccupations communs: «C'est par l'attachement à un territoire, la présence d'un leader, un sentiment d'appartenance au groupe, des règles partagées, des valeurs communes, des langages particuliers, que peut prendre vie une communauté virtuelle $»^{19}$.

Ainsi, pour échapper au flou qui entoure la notion de «communauté virtuelle», nous ne retiendrons, pour comparer les pratiques qui émergent sur la Toile à celles de la kula, que deux exemples: les logiciels libres et l'encyclopédie Wikipédia. L'un et l'autre nous paraissent emblématiques de ces nouveaux «biens communs » en voie de constitution qui, dans un univers largement dominé par le système marchand, ouvrent des espaces de coopération et de gratuité. Certes, ils ne sont pas les seuls ${ }^{20}$, mais ils nous permettent de fonder notre propos sur des pratiques concrètes qu'il s'agit de décrire brièvement.

Qu'est-ce qu'un logiciel libre? Il s'agit de la liberté, pour quiconque, d'utiliser un logiciel, de l'adapter à ses besoins, de le distribuer à d'autres et d'y apporter des améliorations qui pourront profiter à tous ${ }^{21}$. Pour reprendre la comparaison faite par l'inventeur du terme, Richard Stallmann, un logiciel libre est semblable à une recette de cuisine dont on aurait eu connaissance et que l'on peut ensuite diffuser, modifier, et améliorer à sa guise alors que la recette obtenue selon le principe du «logiciel propriétaire » fait l'objet d'une licence d'utilisation, valable dans une seule cuisine, pour un seul utilisateur, et ne peut être ni divulguée ni modifiée (d'autant plus que les «ingrédients de base»-le code source - ne sont pas en libre accès). L'encyclopédie Wikipédia ${ }^{22}$ applique des principes similaires, mais sur le

${ }^{18}$ Pour une typologie de ces divers «environnements sociotechniques de communication en ligne», cf. Serge Proulx, «Les communautés virtuelles construisent-elles du lien social?» (Colloque international sur «L'organisation média. Dispositifs médiatiques, sémiotiques et de médiations de l'organisation », Université Jean Moulin, Lyon, 19-20 novembre 2004), p. 5-6.

19 Jean-François Marcotte, «Communautés virtuelles: la formation et le maintien des groupes sur Internet», Esprit critique, vol. 3, n 10, octobre 2001, <http://vcampus.univ.perp.fr/espritcritique/ 0310/article5.htlm>, p. 2. L'importance de ces règles est également soulignée par Michel Gensollen, art. cité, p. 187-188.

${ }^{20}$ On pourrait mentionner aussi les médias coopératifs (Slashdot.org, Indymedia.org), les publications scientifiques ouvertes, la pratique du copyleft, ou encore des initiatives telles que le WELL (Whole Earth 'Lectronic Link). Cf. Philippe Aigrain, Cause commune. L'information entre bien commun et propriété, Paris, Fayard, 2005, p. 26.

${ }^{21}$ Les logiciels libres sont protégés par le copyleft, c'est-à-dire que leur concepteur est clairement identifié mais qu'il abandonne les droits qui lui seraient normalement acquis tout en se protégeant contre une réappropriation marchande de son invention. La liberté d'usage est donc plus importante que la gratuité.

${ }^{22}$ «Wiki » est le nom d'un logiciel libre (qui signifie «rapide» ou «informel» en hawaïen). Le Temps, 6 septembre 2005. 
plan du contenu plutôt que celui de la technique: chacun peut y accéder librement et gratuitement et peut également modifier la teneur des textes offerts à la consultation; il s'agit donc d'une sorte d'œuvre collective, lancée en 2001, rédigée en 80 langues, qui comprend plus de 700'000 articles en anglais et 150'000 en français, et qui reçoit 80 millions de visites par jour. On lui a évidemment reproché certaines erreurs factuelles, mais celles-ci sont rapidement corrigées par les contributeurs, tous bénévoles, qui doivent respecter des règles de fonctionnement et se soumettre au verdict des «administrateurs » (system operators) qui peuvent, par exemple, décider de supprimer telle ou telle page.

Bien que sommaire, cette présentation suffit sans doute à proposer quelques parallèles avec les remarques faites ci-dessus à propos de la kula:

Premièrement, même si la plupart des grands textes internationaux consacrés aux NTIC privilégient la notion de «libre circulation» de l'information ${ }^{23}$, on ne constate pas, dans les deux exemples retenus, de véritable circulation, dans le sens strict qui caractérise la kula. Toutefois, on peut penser que celui qui propose un logiciel libre ou un article de l'encyclopédie s'attend à ce que quelqu'un d'autre s'y intéresse et contribue à l'améliorer. L'échange peut donc être considéré comme le principe du système. Plus encore, il faut admettre à notre avis qu'il s'agit là d'un échange de dons, à condition de se rappeler que celui-ci est à la fois libre et gratuit mais aussi contraint et intéressé ${ }^{24}$.

Deuxièmement, cet échange accroît la valeur des biens informationnels, notamment parce que ceux-ci sont modifiés au fil du temps (et ne disparaissent pas dans l'acte de «consommation»). Cela dit, l'échange est créateur de prestige, d'abord pour le concepteur qui a la satisfaction de savoir que d'autres se sont intéressés à son travail - et plus ceux-ci seront nombreux, plus ce prestige sera grand -, mais aussi, dans le cadre d'un logiciel libre, pour la marque qui, déposée sous licence GNU ${ }^{25}$, peut acquérir une certaine renommée, associée à celui qui en est l'auteur.

Troisièmement, ces biens informationnels constituent une forme de biens publics, qui sont à la fois non rivaux (ils peuvent être utilisés gratuitement de nombreuses fois ou simultanément par plusieurs personnes) et non exclusifs (puisqu'il est impossible d'empêcher quiconque de se les approprier). Il s'agit donc bien d'une propriété collective, que personne ne contrôle et qui est à la disposition de ceux qui souhaitent en profiter, à l'image des commons.

Quatrièmement, ces échanges sont asynchrones, puisque les réactions des «coopérateurs » (à un logiciel libre ou à un article de l'encyclopédie) prennent

${ }^{23}$ L'anglais est moins précis avec la notion de free flow of information.

${ }^{24}$ Marcel Mauss, «Essai sur le don», Sociologie et anthropologie, Paris, PUF, 1983 [1922], p. 147. Nous nous écartons donc résolument de Michel Gensollen (art. cité) qui, dans sa comparaison entre les échanges de biens informationnels et la kula, ne tient pas compte de cette ambiguïté et ne privilégie que l'intérêt.

${ }^{25}$ Sans entrer dans les détails, GNU est un acronyme récursif (qui se prononce «gnou») et qui signifie «Gnou is Not Unix» (même s'il est compatible avec UNIX!). De même LINUX signifie «Linux Is Not UniX». Cf. Jeremy Blampain et Liliane Palut, Résistance sur Internet. Utopie technologique contre logique marchande, Paris, L'Harmattan, 2000, p. 72 et ss. Plus de 1'000 «développeurs»/informaticiens ont contribué au succès de Linux (un substitut possible de Windows ou $\operatorname{Mac} O S)$. 
nécessairement le temps de la réflexion. Même si les réactions sont parfois rapides (notamment dans le cas de Wikipédia), elle ne sauraient être immédiates.

Cinquièmement, ces biens informationnels échappent à la marchandisation. On se trouve ici au cour de la revendication des «internautes libertaires» qui luttent avec la dernière énergie contre l'appropriation privative de biens informationnels aux rendements croissants (parce que de plus en plus de gens en sont dépendants) et qui critiquent violemment les industries qui en tirent des profits ${ }^{26}$. Alors que, chez les Trobriandais, la distinction entre biens de prestige (vaygu'a) et biens de consommation courante (gimwali) était claire, les biens informationnels sont constamment menacés de passer sous la domination du marché, qui cherche à transformer en biens rivaux ceux qui ne le sont pas $^{27}$.

Sixièmement, on assiste à la création de lien social. Ce qui ne signifie pas que tous les acteurs impliqués dans l'échange partagent les aléas de leur vie quotidienne. Mais, à l'image des chefs rassemblés par la passion de la kula, on retrouve sur la Toile une proximité, ou une complicité, qui déborde les frontières nationales, linguistiques ou culturelles. Contrairement à ce qui se passait dans la kula, ces différents acteurs ne se rencontrent peut-être jamais, mais ils se retrouvent unis par une même volonté de coopération. Leur objectif commun suffit sans doute à créer entre eux des liens plus étroits qu'on ne saurait le penser, même si, pour ne pas perdre la face, ils doivent «tenir leur rang» en contrôlant la qualité de leurs contributions.

\section{LES «AUTRES» NE SONT PLUS CE QU'ILS ÉTAIENT}

Il existe donc, indubitablement, des ressemblances et des différences, entre les échanges caractéristiques de la kula et ceux que l'on peut identifier en examinant une (infime) partie de ceux que l'on retrouve sur la Toile. L'intérêt de Gérald Berthoud pour ces deux univers serait-il donc fortuit? Aurait-il repéré entre eux les similitudes que nous avons soulignées? Aurait-il pensé au contraire que les différences qui les séparent étaient si grandes qu'il fallait de toute urgence s'attaquer à l'engouement généralisé pour la société de l'information, et la marchandisation qui l'accompagne, pour affirmer - avec plus de force encore - le paradigme du don?

Le troisième cas de figure semble le plus plausible. Ainsi, à supposer que Gérald Berthoud ait repéré ces similitudes, ce serait probablement pour en réfuter la proximité réelle et en exposer le caractère illusoire. En effet, en proposant des

${ }^{26}$ Cf. Philippe Aigrain, Cause commune, op. cit., qui inclut - avec raison - dans ses critiques non seulement les logiciels propriétaires, mais aussi les industries agro-alimentaires, pharmaceutiques, etc. La «société de l'information» ne se réduit pas à ce que l'on peut découvrir sur Internet. Voir aussi Bernard Lang, «La bataille des logiciels libres», Annuaire suisse de politique de développement, Genève, IUED, 2003, p. 189-195.

27 «Les industriels de l'information cherchent à définir des supports dont l'information s'évaporerait à la première utilisation, interdisant ainsi à la fois la liberté d'usage, le stockage privé, la copie, l'analyse, le réemploi.», Michel Gensollen, art. cité, p. 196. 
analogies entre la kula des Trobriandais et certaines pratiques repérées sur le réseau des réseaux, nous sommes bien conscients d'avoir pratiqué un exercice de grand écart plutôt acrobatique! Celui-ci cherche d'abord à montrer l'utilité heuristique d'une démarche «d'éclairage en retour» sur nos habitudes d'homo zappiens plutôt que de prouver la présence de l'invariant anthropologique de la réciprocité, qui se manifesterait aujourd'hui dans quelque recoin du champ des nouvelles technologies de l'information. Nous avons cependant jugé utile, voire nécessaire, d'opposer et de rapprocher les deux champs - quand faire se pouvait en privilégiant les pratiques du don, et notamment dans le domaine de l'information digitalisée, pour repenser les deux situations et mener une réflexion qui dépasse la simple opposition. Il s'agissait de montrer que ces deux sociétés ne sont pas totalement irréductibles l'une à l'autre. De toute façon, Gérald s'est peu intéressé aux usages positifs d'Internet ou du marché. Pourquoi? On peut se le demander... et lui suggérer de s'interroger à ce propos.

Cela dit, nous quittons ce chemin de traverse pour rejoindre Gérald Berthoud dans son dépit aggravé face aux avancées de la science occidentale en général et des NTIC en particulier. Non pas simplement pour nous rallier idéologiquement à une position radicalement critique, mais pour nous interroger: pourquoi tant de hargne à l'égard du paradigme du «développement» sous sa forme emblématique du tout marché, et tant de fascination, voire de révérence pour le paradigme du don et les sociétés qui s'en inspirent? La question peut sembler indiscrète, parce qu'elle se situe en quelque sorte hors du champ académique. Or, dans le cadre d'une contribution pour un recueil consacré à un auteur et à un enseignant, il conviendrait de montrer la constance, la cohérence d'un ou de plusieurs intérêts de recherche déployés sur nombre d'ouvrages, d'articles et d'enseignements, de faire état des bifurcations, des moments charnières d'un itinéraire intellectuel qui lui valent l'hommage rendu, ou encore de mettre en avant les nouvelles pistes ou interprétations possibles que suscitent ses travaux.

On pourrait pourtant - à partir de cette question un peu décalée, qui concerne la véhémence de notre collègue à critiquer sa propre société - proposer quelques éléments d'une réflexion qui porte à la fois sur le don et le marché, deux thèmes qui ont toujours été pour lui des sujets emblématiques. Cette réflexion ne s'adresse pas à lui en particulier, mais à l'ensemble dont il fait socialement partie (et nous avec lui), composé de ces personnes, anthropologues ou non, intellectuels ou militants, voyageurs de l'intérieur et explorateurs des lointains, qui se sont appuyées sur «ces gens d'ailleurs » et leurs drôles de manières pour se repenser et pour se regarder «de l'extérieur». Pour elles, ce retournement ou cette découverte ont tenu lieu de rite de passage cognitif dans un autre monde. Après quoi leur propre société leur est apparue pour toujours à la fois familière et étrange, parfois aussi étrangère, voire insupportable. Et les voici désormais munies d'une paire de lunettes à double foyer.

L'ailleurs comme catégorie générale et vague ne suffit pas à tout expliquer. Mais c'est au contact de cette altérité que le paradigme du don, le bien nommé «fait social total», potentialise la force indéfiniment renouvelée de la critique du marché bien sûr et, par extension, oblige à s'opposer à tout ce que ce marché prétend conquérir et/ou détruire. L'ailleurs n'est pas nécessairement géographiquement lointain: il représente ce que nous ne comprenons pas ou ce à quoi nous n'avons pas accès. Il peut également figurer ce qui a été perdu. 
Ce qui rend la critique de la société de marché d'autant plus nécessaire et difficile, c'est que cette perte est camouflée par la prolifération de substituts censés la combler, ce qui empêche de prendre conscience des pertes qu'elle entraîne. Par exemple, le passage d'une communication de type transitif (lorsque l'on communique quelque chose à - ou avec - quelqu'un) à une orgie de «communications » sans objet, révélée par l'usage du verbe «communiquer» dans sa forme intransitive («il communique bien», «il nous faut quelqu'un qui sache communiquer», «la communication est indispensable à l'entreprise», etc.) illustre cette perte de substance, cette hémorragie du sens. On passe ainsi de «l'inter», qui suppose un vis-à-vis, donc une relation sociale qualitative, à «l'hyper», fixé sur le quantitatif. La circularité de la réciprocité, faite de dissymétries alternées ${ }^{28}$ entre les sujets et qui exige du temps, est remplacée par les multiples branchements des machines entre elles qui, tout en écrasant les hommes sur leurs autoroutes de l'information, assurent une équivalence commutative et des hypertextes infinis.

Cela dit, il est peut-être nécessaire de nous interroger sur les différentes formes de l'altérité à partir desquelles nous pourrions fonder une critique de la société. L'anthropologie a jusqu'ici privilégié le recours aux «sauvages». Est-ce la seule voie possible? Même si celle-ci s'est révélée féconde, nous ne pensons pas qu'elle doive constituer l'unique point de repère. Peut-être devrions-nous être attentifs aux différentes formes d'altérité que nous côtoyons, et que nous avons sans doute trop tendance à ignorer. On peut en discerner au moins trois. La première est constituée par un nouveau type d'êtres humains, les hyper-modernes qui nomadisent sur la Toile, ne s'intéressent qu'à eux-mêmes et ne cherchent à «faire société » avec personne. Il est loisible de les critiquer et de refuser de rejoindre leur camp, mais ils existent, et constituent pour beaucoup une sorte de «référence». La deuxième comprend ces «autres de l'intérieur» qui sont rejetés par le système économique ou les normes sociales (les «sans »: sans travail, sans domicile fixe, sans revenu régulier) et auxquels on peut ajouter les paysans - qui ne se considèrent pas comme des «agriculteurs». Enfin, il y a ceux qui résistent, comme les adeptes de la «décroissance», qui s'efforcent de sortir de la société marchande et qui - plus nombreux qu'on ne le croit ${ }^{29}-$ s'efforcent de vivre autrement. Il y aurait ainsi trois figures de l'altérité dans notre propre société: l'altérité figurée par les hypermodernes, une altérité subie (sous l'effet du capitalisme) et une altérité de résistance. Pourquoi ne pas partir de ces trois «types» pour interroger le monde dans lequel nous vivons?

Le sentiment d'inquiétude que distille notre société de marché peut être combattu grâce à la critique, mais celle-ci comporte le risque de préférer à ces « autres » dangereusement proches les autres «classiques » chers aux culturalistes. La réconfortante étrangeté «classique», si bien représentée par le paradigme du don «sauvage», s'est brisée en de multiples fragments. Tout se passe encore comme si, après avoir subi le rite de passage cognitif et affectif mentionné plus

${ }^{28}$ Cette expression est empruntée à Marcel Hénaff, «Remarques sur la Règle d’Or: Ricœur et la question de la réciprocité», in M. Revault d'Allones et Fr. Azouvi (dir.), Ricœur, Paris, Editions de 1'Herne, 2004, p. 336.

${ }^{29}$ Camille Madelain, Pratiques de la décroissance. Itinéraires, Notes et Travaux, $\mathrm{n}^{\circ}$ 76, Genève, Institut universitaire d'études du développement, 2005. 
haut, les anthropologues devaient critiquer la société de marché pour offrir une sorte de contre don à ceux qui, maîtres non conscients du rituel, leur avaient ouvert les yeux. Comme si l'adoption symbolique de l'autre nous avait conféré le droit et l'obligation, en nous appuyant sur eux, de pourfendre les modernes que nous sommes volontairement ou non.

Sans pour autant s'ensauvager ni devenir native, l'anthropologue donne, reçoit, échange une partie de lui-même avec ses interlocuteurs sur le terrain: c'est sa dette souterraine, là où le don rejoint la dette, et la perte, intime, d'une partie dont il ne connaîtra jamais le montant exact - de sa rationalité culturelle de moderne. Il aura ainsi appris qu'il n'a jamais été entièrement moderne. Certes, il se croit et se comporte encore comme un héritier des Lumières (il faut bien enseigner et écrire!), mais une faille s'est introduite dans sa rationalité. Il vit de cette faille, tout en sachant qu'il ne peut renoncer à la rationalité transmise par les siens. Son effort de critique de la société de marché, du «développement», de l'idée de progrès ou de la société de l'information se fera l'écho de cette «scène primitive $»^{30}$ réunissant le rationnel et l'irrationnel, à laquelle il aura assisté ou participé, et qui les mêlera indistinctement jusqu'à lui faire récuser à tout jamais la théorie du «grand partage».

Cet événement fondateur d'un regard, donc d'une épistémologie et d'une éthique, est, en ce qui concerne Gérald Berthoud, une fiction biographique, mais c'est ce qui se passe bien souvent pour l'anthropologue ou plus généralement pour tout $«$ exote $»^{31}$. Toutes ces personnes qui pratiquent une réflexivité appuyée sur une ou des altérités (au sens large) sont en route vers un ailleurs recomposé au fur et à mesure de leurs itinéraires et de leurs errances. En route, mais aussi «entre deux »: elles ne sont plus des Argonautes mais, sans être déjà des Hypernautes, elles sont bon gré mal gré déjà des Internautes. Les premières sont toujours à la recherche de la Toison d'Or ou d'un vagyu'a qui satisfasse leur quête, les deuxièmes sont des croyants, adeptes de la religion du savoir digitalisé en vogue dans la société de l'information (mais peut-être aussi séduits par la nécessité du don), et les troisièmes sont de simples usagers d'Internet, sceptiques et critiques. Entre elles, la différence est considérable... et les similitudes plus grandes qu'on ne peut l'imaginer. Gérald Berthoud s'est intéressé à tous et son parcours intellectuel montre que, finalement, les uns et les autres sont plus proches qu'il ne l'a luimême pensé.

Institut universitaire d'études du développement, Genève

Marie-Dominique.Perrot@iued.unige.ch

Gilbert.Rist@iued.unige.ch

${ }^{30}$ L'expression ici ne se réfère pas littéralement au concept freudien mais lui donne le sens métaphorique qui désigne un événement fondamental. L'événement premier du choc de la rencontre se répercutera, tout au long de l'existence et à la suite d'autres rencontres, comme autant de ricochets de la première. Elle souligne aussi que le terrain de l'anthropologue et son rapport aux indigènes restera toujours une boîte noire érigée en mythe.

${ }^{31}$ Victor Segalen, Essai sur l'exotisme: une esthétique du divers, Paris, Fata Morgana, 1978. 